\title{
Phase Behavior of Ternary Blends of Tactic Poly(methyl methacrylate)s and Poly(vinylidene chloride-co-acrylonitrile)
}

\author{
Wen-Ping $\mathrm{HSU}^{\dagger}$ \\ Department of Applied Chemistry, Chia-Nan University of Pharmacy and Science, \\ \#60 Sec. 1 Erh-Jen Road, Jen-Te Hsian, Tainan, Taiwan, Republic of China 71710
}

(Received March 9, 2000; Accepted June 13, 2000)

\begin{abstract}
Previously, isotactic and atactic poly(methyl methacrylate)s (PMMAs) were found to be miscible with poly(vinylidene chloride-co-acrylonitrile) (Saran F) because all the prepared films were transparent and showed composition dependent glass transition temperatures $\left(T_{\mathrm{g}} \mathrm{s}\right)$. However, the syndiotactic PMMA/Saran F blends were immiscible because most of the cast films were translucent and had two glass transition temperatures. Atactic PMMA is known to be miscible with syndiotactic PMMA according to literature. So atactic PMMA is miscible with both Saran F and syndiotactic PMMA but Saran F and syndiotactic PMMA are immiscible. It will be interesting to study the miscibility of ternary blends of atactic and syndiotactic PMMAs and Saran F. Therefore an investigation based on this is reported in this article. Calorimetry was used as the principal tool to study miscibility. A blend composed of atactic and syndiotactic PMMAs was prepared previously and confirmed to be miscible. An approximate phase diagram of the ternary blends was established based on differential scanning calorimetry data. The results indicated that for all the studied compositions, the ternary blends were determined to be miscible. Most of the blend $T_{\mathrm{g}}$ values were fitted well by the Fox equation. For the syndiotactic PMMA/Saran F(wt ratio=1) blend, adding of at least 12.5 wt\% of atactic PMMA was sufficient to cause the immiscible blends to be miscible.

KEY WORDS Tacticity/Poly(methyl methacrylate)/Poly(vinylidene chloride-co-acrylonitrile)/'Ternary Blends /
\end{abstract}

Ternary blends are gaining importance in the field of polymers through years. The first systematic study on ternary blends was reported by Kwei et $a l^{1}$ in 1977 . In their study, the addition of poly(vinylidene fluoride) (PVDF) to the immiscible pair PMMA/poly(ethyl methacrylate) (PEMA) was studied and found to be miscible. A list of ternaries investigated has been considerably enlarged since then..$^{-8}$ In nearly all these blends, a third component either a homopolymer or copolymer is added to homogenize an immiscible pair. Miscibility is often achieved in cases where this third component is miscible with other polymers.

Recently, Bicakci and Cakmak ${ }^{9}$ investigated the phase behavior of binary and ternary blends of poly(ethylene naphthalate) (PEN), poly(ether imide) (PEI) and poly(ether ether ketone) (PEEK) using differential scanning calorimetry (DSC) and dynamic mechanical analysis (DMTA) techniques. PEN/PEI and PEI/PEEK binary blends exhibit single glass transition temperatures in full composition range and PEN and PEEK were found immiscible particularly at mid-concentrations.

When PEI is added to the immiscible PEN/PEEK system, first the blends form two separated PEN-rich and PEEK-rich phases below about $40 \%$ PEI concentration, and above this concentration, the three homopolymers form a miscible phase in the amorphous state exhibiting a single $T_{\mathrm{g}}$. An approximate ternary phase diagram was established by them based on the DSC and DMTA results.

In a previous study, ${ }^{10}$ isotactic, atactic and syndiotactic PMMAs (designated as i, a and sPMMAs) with approximately the same molecular weight were blended with poly(vinylidene chloride-co-acrylonitrile) (Saran F) (containing $20 \mathrm{wt} \%$ of acrylonitrile) in tetrahydrofuran

\footnotetext{
${ }^{\dagger}$ Fax: $+886-6-2667319$
}

to cast into films. The glass transition temperatures of the polymers were measured calorimetrically. iPMMA and aPMMA were found miscible with Saran $F$ because all the prepared films were transparent and had a single composition dependent glass transition temperature $\left(T_{\mathrm{g}}\right)$. However, sPMMA is not miscible with Saran F because most of the cast films were translucent and had two $T_{\mathrm{g}} \mathrm{s}$. Since sPMMA is miscible with aPMMA according to the literature ${ }^{10}$ but immiscible with Saran $\mathbf{F}$ and aPMMA is miscible with Saran F. Therefore an investigation of the miscibility of ternary blends composed of aPMMA, sPMMA and Saran F is worthwhile and was pursued in this laboratory. aPMMA is acting as a "cosolvent" between sPMMA and Saran F in this study.

In this article, the ternary blends of aPMMA, sPMMA and Saran F were prepared in several weight ratios. The glass transition temperatures of the ternary mixtures were determined calorimetrically. An approximate phase diagram of the ternary blends was established based on calorimetry data and a single $T_{\mathrm{g}}$ was used as the criterion for determining miscibility. The results indicated that the ternary blends were miscible for all the studied compositions.

\section{EXPERIMENTAL}

\section{Materials}

Atactic and syndiotactic PMMAs (designated as a and sPMMAs in this study) were purchased from Polysciences, Inc., Warrington, PA. According to supplier information, the molecular weights $\left(M_{\mathrm{w}} \mathrm{s}\right)$ of aPMMA and sPMMA are the same about 100000 . The estimation of meso $(\mathrm{m})$ and racemic ( $\mathrm{r}$ ) fractions was reported previously. ${ }^{11}$ The calculated $\mathrm{m}$ and $\mathrm{r}$ fractions of aPMMA and sPMMA are $33.8 \%$ and $66.2 \%$ and $9.3 \%$ and $90.7 \%$, respectively. The error is about 5 to $8 \%$. The (vinylidene 
Table I. Glass transition temperatures of ternary blends

\begin{tabular}{lrrrr} 
Saran F/aPMMA & $T_{\mathrm{gsc}} /{ }^{\circ} \mathrm{C}$ & $T_{\mathrm{gq}} /{ }^{\circ} \mathrm{C}$ & $T_{\mathrm{g}}{ }^{\mathrm{a}}{ }^{\circ} \mathrm{C}$ & $\Delta T_{\mathrm{gq}} /{ }^{\circ} \mathrm{C}$ \\
\cline { 1 - 3 } 1. $(12.6 / 12.5 / 74.9)$ & 101 & 110 & 110 & 20 \\
$2 .(12.5 / 37.5 / 50.0)$ & 96 & 106 & 106 & 18 \\
$3 .(12.6 / 62.5 / 24.9)$ & -- & 99 & 101 & 15 \\
$4 .(12.4 / 75.1 / 12.5)$ & 91 & 102 & 99 & 18 \\
$5 .(25.1 / 37.3 / 37.6)$ & 101 & 97 & 98 & 22 \\
$6 .(25.0 / 50.0 / 25.0)$ & 102 & 96 & 95 & 17 \\
$7 .(25.1 / 56.2 / 18.7)$ & 88 & 96 & 94 & 19 \\
$8 .(26.4 / 19.9 / 53.7)$ & 74,94 & 101 & 100 & 22 \\
$9 .(37.5 / 25.0 / 37.5)$ & 81,123 & 93 & 92 & 22 \\
$10 .(43.75 / 12.5 / 43.75)$ & --- & 111 & 90 & 8 \\
$11 .(50.1 / 12.5 / 37.4)^{\mathrm{b}}$ & 71,94 & 90 & 81 & 19 \\
$12 .(49.9 / 25.2 / 24.9)^{\mathrm{b}}$ & 67,92 & 90 & 84 & 27 \\
$13 .(50.0 / 37.5 / 12.5)^{\mathrm{b}}$ & 70,94 & 73 & 86 & 24 \\
$14 .(75.0 / 12.5 / 12.5)$ & 76 & 81 & 71 & 16
\end{tabular}

${ }^{a}$ Estimated from the Fox equation. ${ }^{\mathrm{b}}$ Taken from a previous publication. ${ }^{10}$

chloride-co-acrylonitrile) copolymer (Saran F) used for this study contained $20 \mathrm{wt} \%$ acrylonitrile (AN) units and was also obtained from Polysciences. The $M_{\mathrm{w}}$ value for Saran F is approximately 260000.

\section{Film Preparation}

Thin films of ternary blends of aPMMA, sPMMA and Saran $\mathrm{F}$ in different weight ratios were made by solution casting onto glass plates. The actual compositions of the ternary blends are shown in Table I. Tetrahydrofuran (THF) was used as solvent for all the blend compositions. THF is A.C.S. reagent purchased from Fisher Scientific, Fair Lawn, New Jersy. Because Saran F and its blends start to decompose at around $104-116^{\circ} \mathrm{C}$, therefore the final drying step for all the films took place in a vacuum oven at $75^{\circ} \mathrm{C}$ for $15-23 \mathrm{~h}$ to avoid film degradation. Then the films were cooled to room temperature slowly by air to make as-cast samples. The as-cast samples were used for DSC studies.

\section{Differential Scanning Calorimetry (DSC)}

Glass transition temperatures $\left(T_{\mathrm{g}} \mathrm{s}\right)$ of the polymer blends were determined by a DuPont 2000 thermal analyzer. According to our experimental results, Saran F had a higher thermal stability in nitrogen environment than in vacuum. In repetitive test runs, we found that Saran F still maintained its thermal stability when the temperature was as high as $200^{\circ} \mathrm{C}$. Therefore the experiments were performed in two consecutive scans from 30 to $200^{\circ} \mathrm{C}$ in the ambient environment of nitrogen gas at a flowing rate of $100-110 \mathrm{~mL} \mathrm{~min}^{-1}$. At the end of the first thermal scan, the samples stayed at $200^{\circ} \mathrm{C}$ for one minute. The samples were then quenched to $0^{\circ} \mathrm{C}$ immediately using an ice-water bath and were scanned the second time. A heating rate of $20^{\circ} \mathrm{C} \mathrm{min}{ }^{-1}$ was used in each scan. Based on the observation, the annealing time was enough for the determination of $T_{\mathrm{g}}$. The samples were quenched quickly to prevent crystallization as detected by DSC There was no trace of solvent in the films detectable by DSC The inflection point of the specific heat jump of a thermal scan was taken as the glass transition temperature. The glass transition temperatures determined from the first and second thermal scans were designated as $T_{\mathrm{gsc}}$ and $T_{\mathrm{gq}}$, respectively. Therefore,

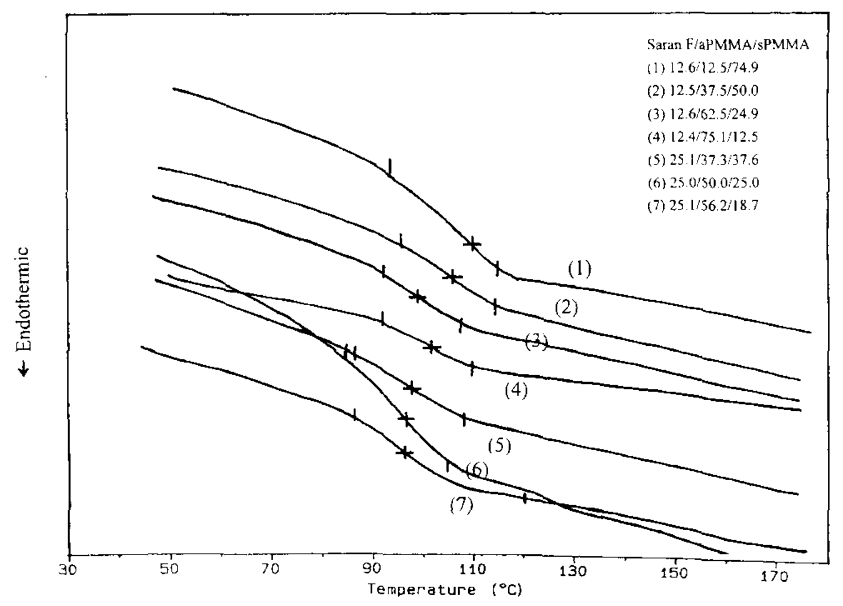

Figure 1. DSC thermograms of the ternary blends(\#1-\#7).

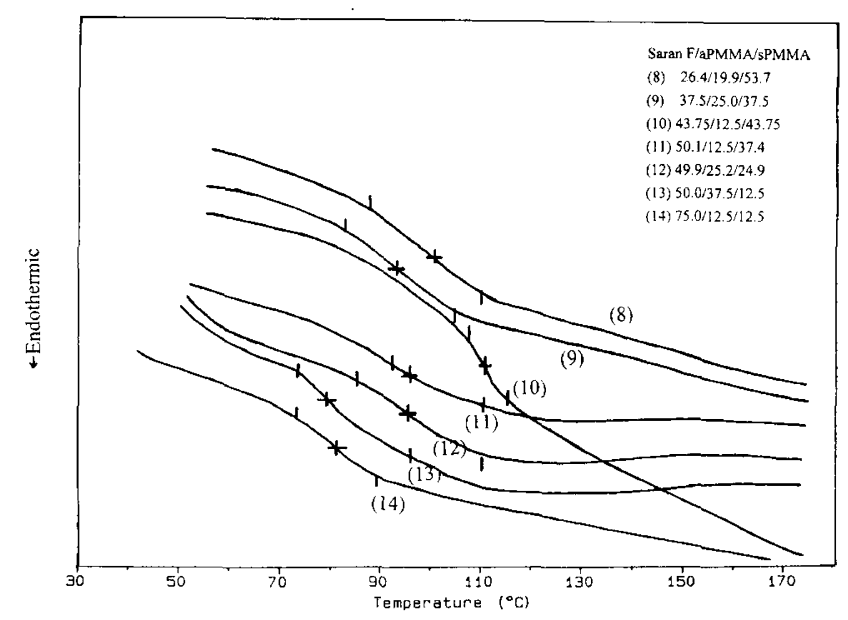

Figure 2. DSC thermograms of the ternary blends(\#8-\#14).

$T_{\mathrm{gsc}}$ is the $T_{\mathrm{g}}$ of the slowly cooled (as-cast) films and $T_{\mathrm{gq}}$ that of the quenched films.

\section{RESULTS AND DISCUSSION}

\section{Glass Transition Temperatures}

For representation, only the second thermal scans of the ternary blends are shown in Figures 1 and 2, respectively. Figure 1 presents thermal scans of the ternary blends containing $c a .12 .5$ or $25.0 \mathrm{wt} \%$ Saran F. Thermal scans of the ternary blends containing more than $25.0 \mathrm{wt} \%$ Saran F are given in Figure 2. Obviously all the studied ternary blends in Figures 1 and 2 showed one $T_{\mathrm{g}}$. The estimated $T_{\mathrm{gsc}}$ and $T_{\mathrm{gq}}$ values of the ternary blends from the first (not shown) and second scans are listed in Table I. For several blend compositions especially those containing more than $25 \mathrm{wt} \%$ Saran F, there is difference between the $T_{\mathrm{gsc}}$ and $T_{\mathrm{gq}}$ values. Because the $T_{\mathrm{gq}}$ values are considered to be free of annealing or other thermal/solvent effects, the ternary blends are determined to be miscible based on a single $T_{\mathrm{gq}}$ value. Because slow-cooled \#8 to \#13 samples have two $T_{\mathrm{g}} \mathrm{s}$ first, then after quenching all these samples show one $T_{\mathrm{g}}$. Therefore thermodynamically speaking, they are miscible at $200^{\circ} \mathrm{C}$ but immiscible at room temperature. The 
glass transition temperature regions $\left(\Delta T_{\mathrm{gq}}\right)$ (shown as the last column in Table I) were calculated as differences between the onset and end points of $T_{\mathrm{gq}} . \Delta T_{\mathrm{gq}}$ does show broadening in most of the compositions and this is quite common in the multi-component system.

\section{Fox and Other Equations}

For polymer blends with weak or no interaction, the Fox equation ${ }^{12}$ seems to predict the glass transition temperature quite well. The Fox equation extended for a ternary mixture is shown as below in eq 1

$$
1 / T_{\mathrm{g}}=w_{1} / T_{\mathrm{g} 1}+w_{2} / T_{\mathrm{g} 2}+w_{3} / T_{\mathrm{g} 3}
$$

where $T_{\mathrm{g}}$ is the glass transition temperature of a blend, $T_{\mathrm{gi}}$ and $w_{\mathrm{i}}$ are the glass transition temperature and the weight fraction of polymers $i$, respectively $(i=1,2,3)$. The $T_{\mathrm{g}}$ values of Saran F, aPMMA and sPMMA were obtained previously ${ }^{10}$ to be $59^{\circ} \mathrm{C}, 103^{\circ} \mathrm{C}$, and $122^{\circ} \mathrm{C}$, respectively. All these values were used in eq 1 to estimate the $T_{\mathrm{g}}$ values of the ternary blends (designated as $T_{\mathrm{g}}^{a}$ in Table I). For the ternary blends containing $37.5 \mathrm{wt} \%$ or less Saran F(\#1 to \#9 blends), the Fox equation seems to predict the experimental $T_{\mathrm{g}}$ quite well. The difference between the Fox equation predictions and experimental $T_{\mathrm{g}}$ values can be seen more clearly in Figure 3. Aside from \#1 to \#9 blends, the Fox equation seems to underestimate the $T_{\mathrm{g}}$ values of most of the rest of the ternary blends (from \#10 to \#14 except \#13).

Attempts were made to fit $T_{\mathrm{g}}$ of \#10 to \#14 blends (\#13 not included) using other equations. The methodology is briefly illustrated as follows: Since the aPMMA/sPMMA blends were observed previously ${ }^{10}$ to have a $T_{\mathrm{g}}$ elevation compared to the additivity rule. The simplified Kwei ${ }^{13}$ equation as in eq 2 was used to fit the $T_{\mathrm{g}}$ values of the aPMMA/sPMMA blends

$$
T_{\mathrm{g}}=w_{1} T_{\mathrm{g} 1}+w_{2} T_{\mathrm{g} 2}+q w_{1} w_{2}
$$

where symbols have the same meanings as in eq 1 and $q$ is a parameter related to the strength of interaction between two polymers. For the aPMMA/sPMMA blends, $q$ was estimated to be 20 . Then the ternary blends were assumed to be a pseudo-binary mixture of Saran F and aPMMA/sPMMA. The $T_{\mathrm{g}}$ values of these pseudo-binary blends were estimated by weight average:

$$
T_{\mathrm{g}}=w_{\mathrm{i}} T_{\mathrm{gi}}+w_{\mathrm{j}} T_{\mathrm{gj}}
$$

For our special case, $T_{\mathrm{g}}, T_{\mathrm{gi}}$, and $T_{\mathrm{gj}}$ are the glass transition temperatures of a ternary blend, Saran $F$ and aPMMA/sPMMA blend, respectively. $w_{\mathrm{i}}$ and $w_{\mathrm{j}}$ are the weight fractions of Saran F and aPMMA/sPMMA blend. The estimated $T_{\mathrm{g}}$ values (designated as $T_{\mathrm{g}}$ ) from eq 3 are tabulated in Table II. The $T_{\mathrm{g}}$ values of the ternary blends containing ca. 50 wt\% Saran F (\#11 and \#12) can be estimated by this method. Take \#11 blend for example. The \#11 blend has the composition of Saran F/aPMMA/sPMMA(50.1/12.5/37.4). So the blend is composed of $50.1 \%$ Saran F and $49.9 \%$ aPMMA/sPMMA. First, the $T_{\mathrm{g}}$ of aPMMA/sPMMA(12.5/37.4 converted to $25.1 / 74.9)$ was estimated from eq 2 to be $121^{\circ} \mathrm{C}$. Then the $T_{\mathrm{g}}$ of blend \#11 was calculated from eq 3 to be $90^{\circ} \mathrm{C}$

Polym. J., Vol. 32, No. 10, 2000

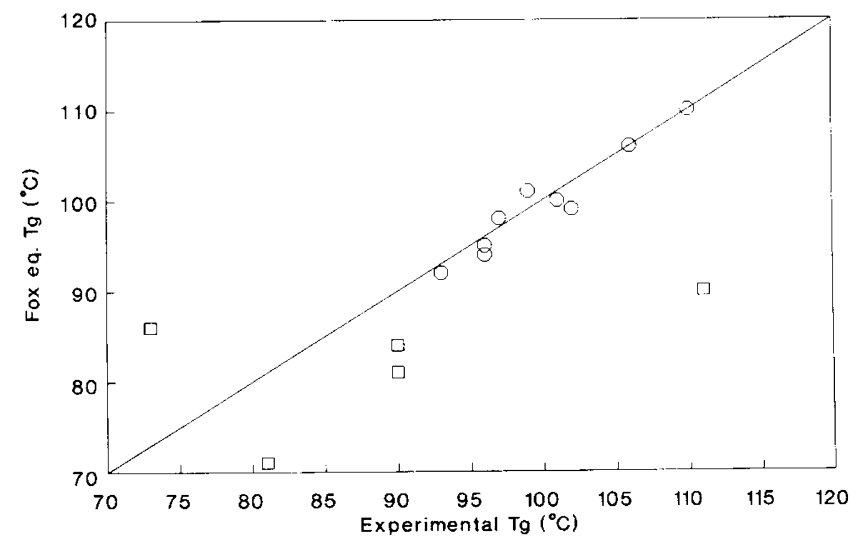

Figure 3. Glass transition temperatures of the ternary blends. $\bigcirc: \# 1$ to $\# 9, \square$ : \#10 to \#14.

Table II. Glass transition temperatures of certain ternary blends

\begin{tabular}{lcc}
\hline Saran F/aPMMA/sPMMA & $T_{\mathrm{gq}} /{ }^{\circ} \mathrm{C}$ & $T_{\mathrm{g}}{ }^{\mathrm{a}} / \mathrm{C}$ \\
\hline $10 .(43.75 / 12.5 / 43.75)$ & 111 & 94 \\
$11 .(50.1 / 12.5 / 37.4)$ & 90 & 90 \\
$12 .(49.9 / 25.2 / 24.9)$ & 90 & 88 \\
$14 .(75.0 / 12.5 / 12.5)$ & 81 & 74 \\
\hline
\end{tabular}

${ }^{a}$ Estimated from the eq 3.

$(=0.501 \times 59+0.499 \times 121)$. However for \#10 and \#14 blends, the agreement between experimental and estimated $T_{\mathrm{g}}$ is not good. The $T_{\mathrm{g}}$ of the \#10 blend is much higher than that estimated either by the Fox equation or eq 3 . For blend \#14, improvement in $T_{\mathrm{g}}$ estimation is seen in the prediction value by eq 3 .

\section{Co-solvent Effect of aPMMA}

For the purpose of illustrating co-solvent effect of aPMMA, the $T_{\mathrm{g}}$ values of the blends with sPMMA/Saran $\mathrm{F}$ wt ratio $=1$ were plotted in Figure 4. Previous results $^{10}$ of the aPMMA/Saran $F$ and aPMMA/sPMMA blends were also presented for comparison. Since the $T_{\mathrm{g}}$ difference between aPMMA/Saran F and aPMMA/ sPMMA blends with the same aPMMA composition is always greater than $20^{\circ} \mathrm{C}$, the single $T_{\mathrm{g}}$ criterion for miscibility seems to be justified in our ternary system. The following comments can be drawn from Figure 4. Firstly, the ternary $T_{\mathrm{g}}$ values are located between those of the two binaries with the same aPMMA composition. Secondly, adding of at least $12.5 \mathrm{wt} \%$ aPMMA into the sPMMA/Saran F mixture is sufficient to cause miscibility. It is interesting that the addition of a small amount of aPMMA produces a single $T_{\mathrm{g}}$ of the ternary blends. The reason may be due to the immiscibility between $\mathrm{Sa}-$ ran $\mathrm{F}$ and sPMMA is not very high. The unusual high $T_{\mathrm{g}}$ behavior of the high $T_{\mathrm{g}}$ phase of sPMMA/Saran F(50/50) blend was explained previously ${ }^{10}$ to be likely due to a highly syndiotactic PMMA presence.

\section{Phase Diagram and Binary Interaction Model}

Using the single $T_{\mathrm{g}}$ as the criterion for the miscibility, the phase diagram of the ternary blends composed of Saran F, aPMMA, and sPMMA is shown in Figure 5. Be- 


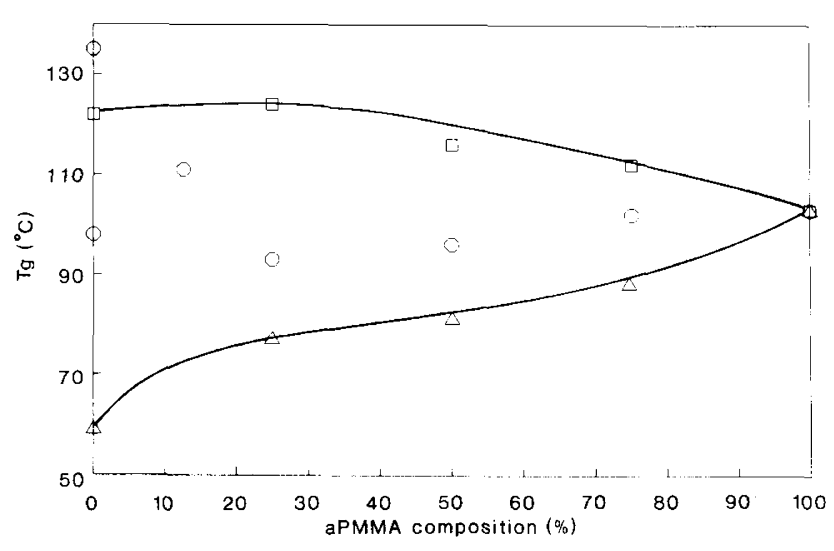

Figure 4. Change of $T_{\mathrm{g}}$ of the ternary blends with aPMMA composition (sPMMA/Saran $\mathbf{F}$ wt ratio=1). $O$ : data point, $\triangle: T_{\mathrm{g}}$ of aPMMA/Saran $F$ blend (curve drawn for viewing), $\square: T_{g}$ of aPMMA/sPMMA blend (curve drawn for viewing).

cause all the studied compositions of the ternary blends are miscible, the only possible immiscible region is the area surrounding the two immiscible sPMMA/Saran F (75.0/25.0 and 50.0/50.0) blends. Therefore it can be concluded that the ternary blends containing SPMMA Saran F wt ratio $\geq 1$ and a very low concentration of aPMMA (definitely lower than $12.5 \%$ according to data) are likely to be immiscible.

A simple model without considering the effect of polymer molecular weight as the following was used to explain the immiscible region of the ternary blends. This model assumes mixing $\Delta H_{\mathrm{m}}$ of a ternary system can be described in terms of binary interaction coefficients:

$$
\Delta H_{\mathrm{m}}=2 R T\left(\chi_{12} \Phi_{1} \Phi_{2}+\chi_{13} \Phi_{1} \Phi_{3}+\chi_{23} \Phi_{2} \Phi_{3}\right)
$$

where $R$ is the gas constant, $T$ the temperature, $\chi_{i j}$ the thermodynamic interaction parameter between $i$ and $j$ (which determines the sign and magnitude of the best of mixing of $i$ with $j$ ) and $\Phi_{i}$ is the volume fraction of component $i$ in the mixture. Equation 4 assumes that the $\chi$ parameters are independent of concentration and symmetrical, i.e., $\chi_{i j}=\chi_{j i}$. For the purpose of the present discussion, eq 4 can be reduced, for measurements carried out at a constant temperature, to:

$$
\chi \approx \chi_{12} \Phi_{1} \Phi_{2}+\chi_{13} \Phi_{1} \Phi_{3}+\chi_{23} \Phi_{2} \Phi_{3}
$$

where $\chi$ is the thermodynamic interaction parameter of the ternary blends.

Equation 5 was then used to calculate miscibility/immiscibility boundary observed in Figure 5. For that purpose, immiscibility was associated with a positive $\chi$ value and miscibility with a negative $\chi$ value. $\chi$ is set equal to zero to calculate concentrations, assuming that a true ternary solution is present in the area where a single $T_{\mathrm{g}}$ is observed. In this calculation, aPMMA, sPMMA, and Saran F were designated as polymers 1, 2, and 3 , respectively. Also the volume fraction was not used but the weight fraction was used tentatively. Since aPMMA(1) is both miscible with $\operatorname{SPMMA}(2)$ and Saran $\mathrm{F}(3)$, therefore their $\chi$ values were set to be negative. The value of $\chi_{13}$, between aPMMA and Saran F,

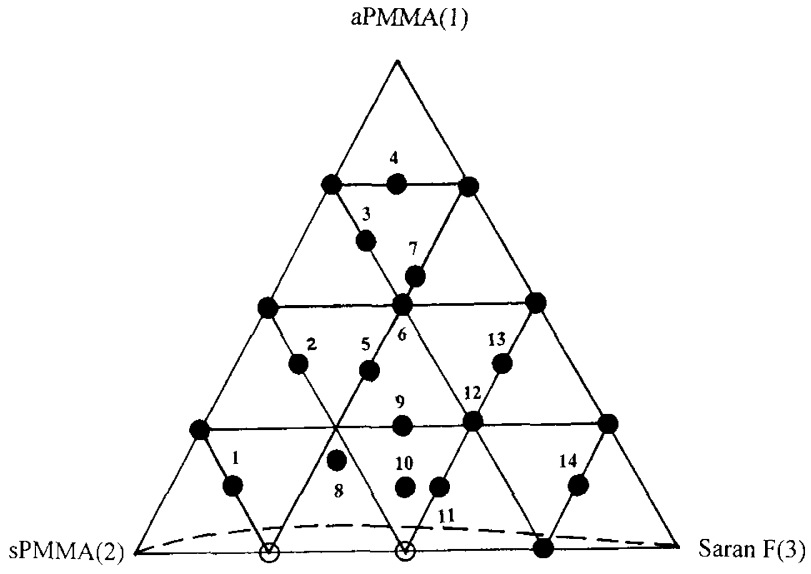

Figure 5. Phase diagram of the ternary blends. $0:$ miscible $\bigcirc$ : immiscible, … : estimated immiscible region, numbers in the figure indicate the same compositions in Table I.

was selected being more negative than $\chi_{12}$; this is a necessary requirement in order to induce an asymmetry to better corner the immiscible region. Finally, $\chi_{23}$, between SPMMA and Saran F, was selected as being positive because of the immiscibility between these two polymers.

Although we don't have data of the absolute $\chi$ values of our ternary system, the following set of $\chi_{i j}$ (ScottTompa) values were used tentatively. $\chi_{12}=-0.003, \chi_{13}$ $=-0.018$, and $\chi_{23}=0.002$. The $\chi_{23}$ value was chosen to be 0.002 about twice $\chi_{\mathrm{c}}$ of Saran F/sPMMA blend. $\chi_{\mathrm{c}}$ was estimated to be about 0.001 based on the following relation $^{14}$

$$
\chi_{\mathrm{c}}=(1 / 2)\left[\left(1 / m_{1}\right)^{0.5}+\left(1 / m_{2}\right)^{0.5}\right]^{2}
$$

where $m_{i}$ is chain length of polymer i. As shown in Figure 5, agreement between model and data is not satisfactory but qualitative trend of the immiscible region can be described. Obviously, this model has some shortcoming. $\chi_{23}$ is concentration dependent based on experimental data. ${ }^{10}$ It was observed that at high concentration of Saran F binary blends of sPMMA and Saran F are miscible. At mid and sPMMA-rich concentrations, there are two distinct $T_{\mathrm{g}}$ values attributable to the blend components and characteristic of an immiscible blend. Therefore the assumption of concentration independence and symmetry is in error. The present approach, with its numerous assumptions and imperfect agreement with the experimental data, cannot be interpreted in a fully quantitative manner. Nevertheless, it certainly indicates favorable interactions in the aPMMA/sPMMA and aPMMA/Saran F blends, and some unfavorable interactions existing between sPMMA and Saran F. Form the predictions of this simple model, it is implied that aPMMA/Saran $F$ interaction is stronger than aPMMA/ sPMMA interaction.

\section{CONCLUSIONS}

aPMMA is both miscible with sPMMA and Saran F. However, sPMMA and Saran F are immiscible. Addition 
of aPMMA into the sPMMA/Saran F mixture causes the ternary blends of Saran F, aPMMA, and sPMMA to be miscible for all the studied compositions. For sPMMA Saran $\mathrm{F}$ (wt ratio $=1$ ) blend, it is interesting to find out adding of a small amount of aPMMA (12.5 wt\%) causes miscibility of the ternary blends. The possible immiscible phase regions are those ternaries containing a very low concentration of aPMMA and a high sPMMA/Saran $F$ ratio. The simple interaction model suggests that favorable interactions exist in the aPMMA/sPMMA and aPMMA/Saran $F$ blends and unfavorable interactions between sPMMA and Saran F. The model also implies that the aPMMA/Saran F interaction is very likely to be stronger than the aPMMA/sPMMA interaction.

Acknowledgment. The financial support by the National Science Council of Taiwan, R.O.C. NSC-88-2216E-041-001 and the fund given by Chia-Nan College of Pharmacy and Science, Grant CNAC-89-05 are greatly appreciated. Thanks are extended to Prof. T. K. Kwei at Polytechnic University, New York for his encouraging discussions.

\section{REFERENCES}

1. T. K. Kwei, H. L. Frisch, W. Radigan, and S. Vogel, Macromolecules, 10, 157 (1977).

2. Y. Y. Wang and S. A. Chen, Polym. Eng. Sci., 21, 47 (1981).

3. D. Rigby, J. L. Lin, and R. J. Roe, Macromolecules, 18, 2269 (1985).

4. V. Shah, J. D. Keitz, D. R. Paul, and J. W. Barlow, J. Appl. Polym. Sci., 32, 3863 (1986).

5. J. I. Equizabal, J. J. Irvin, M. Cotazar, and G. M. Guzman, J. Appl. Polym. Sci., 32, 5945 (1986).

6. S. H. Goh, K. S. Siow, and K. S. Yap, Thermochim. Acta, 105, 191 (1986).

7. S. H. Goh and K. S. Siow, Thermochim. Acta, 102, 281 (1986).

8. W. H. Christiansen, D. R. Paul, and J. W. Barlow, J. Appl. Polym. Sci., 34, 537 (1987).

9. S. Bicakci and M. Cakmak, Polymer, 39, 4001 (1998).

10. W. P. Hsu and C. F. Yeh, J. Appl. Polym. Sci., 75, 1313 (2000).

11. W. P. Hsu and C. F. Yeh, J. Appl. Polym. Sci., 73, 431 (1999) and references therein.

12. T. G. Fox, J. Appl. Bull. Am. Phys. Soc., 1, 123 (1956).

13. T. K. Kwei, J. Polym. Sci., Polym. Lett. Ed., 22, 306 (1984).

14. R. L. Scott, J. Chem. Phys., 17, 279 (1949). 